\title{
FROM BA TO BQA: INCREASING ROLE OF ANALYSTS IN IMPROVING PRODUCT QUALITY IN AGILE ENVIRONMENTS
}

\author{
Faizan Ahmed, Irtaza Tarar and Muhammad Abbas \\ National University of Sciences and Technology (NUST), Islamabad, Pakistan
}

\begin{abstract}
Quality Software is one of the most sought out commodity in the digital world. Different quality standards like GQM, FURPS, ISO 9126 etc. have been proposed over the years and are continued to be used by companies as per their quality requirements, quality goals, kind of products, services and development processes. While traditional development practices place responsibility of Quality goals with Quality Engineers, Testers etc. recently there has been an increase in talks of integrating Business Analysts (BAs) role in defining and validating quality requirements especially in Agile based software developments, so much so as to suggestions of creating a BQA (BA/QA) role; integrating functional responsibilities of two in a single role in Agile frameworks. Proponents of this philosophy backs BAs for this challenging role because of their familiarity with both business and technological needs of clients, as well as knowledge of in-house products and services. Using statistics from a global SAAS based company; this paper provides valuable insights that such roles have started to exist in our Industry with good effect while bringing a positive impact on product quality and ultimately increase in customer satisfaction.
\end{abstract}

\section{KEYWORDS}

BAs (Business Analysts), QAs (Quality Analysts), BQA (Business Quality Analyst), Software as a Service (SaaS), Agile, Scrum, Software Quality

\section{INTRODUCTION}

Forbes defines Subject Matter Experts (SMEs) as among the top 10 business trends that will drive customer success in 2017 and 2018. As more and more companies embrace technology to automate their business processes, SMEs work hand in hand with BAs (at times as BAs) to ensure the delivery of correctly conceived solutions. With IT industry fast moving towards Service based Architecture (SaaS) - commonly via Agile based product centric approach - and Businesses putting high focus on quality and timely delivery, the intermediary role of BAs is evolving into one that has a direct impact on Product Quality. As agents of change, and being in a position where they can understand both business and technical needs of to be developed services, BAs are well placed to identify problems beforehand and highlight key quality matrices, ensuring product quality and clients satisfaction are always met. Starting with a brief overview of existing literature that directly/indirectly calls for such a role (next section), this paper provides a numerical analysis on how a key US based Software company has practically embraced this new BQA position in their Agile environment to improve product maturity and quality, and how other enterprises can follow suit.

\section{NEED FOR A BUSSINGESS QUALITY ANALYST (BQA)}

Feldman (2005) and Vinod (2015) stresses that Quality assurance is not a one-time activity in software projects but it is an ongoing process. During the software development, there is always a miscalculated cost for the unpredicted repeated actions. One of these actions is reopening of the user stories. User stories are reopened due to the errors committed at four major fronts; developers, quality analyst (QA), business analysts (BA) and environment issues. Since a Business analyst (BA) is responsible for analyzing and 
receiving the user requirements and then developing the user stories while a Quality analyst (QA) works as testers of user stories. So if these two positions can be integrated, the reopening of user stories can be avoided to decrease the additional costs faced during the software development cycle.

Daneva et al. (2013) in their exploratory study discuss role of Software Architects (SAs) in dealing with Quality Requirements (QRs) in context of large component based SAAS projects. Their findings reflect that by involving SAs with QRs leads to better definitions, better designs and hence better results in terms of customer satisfaction and product quality. Their study discusses direct impact that SAs have in QRs discovery, documentation, quantification, and in later stages, validation. It stresses that with SAs involvement: QRs are approached the same way as functional and architecture design requirements, ownership of QRs is proactively embraced, quantification is specialized as per specific subfields e.g. response time, availability etc., prioritization is handled keeping costs, values and benefits in focus with help of clients, and QRs validation is taken as a social activity rather than a method-centric checklist process.

Bennett et al. (2013) suggests role revamp of REs by allowing them to handle data, quality and security requirements in addition to their existing requirement gathering role. Quality criteria that they focus include performance, reliability and maintainability requirements. They stress that since requirement changes are an inherent part of RE process, continuous interfacing between customer stakeholders and REs puts them in an ideal place to forecast quality values for clients.

Herrmann et al. (2013) study focuses on existing disparity between the theoretical role of REs and the industry wide practiced role. Based on empirical analysis of job advertisements and interviews conducted from RE practitioners between 2009 - 2012, his study concludes that RE mostly exists as an Ad Hoc role with focus on experience, soft skills and solution design as key characteristics and that REs spend nearly 1/3rd (approx. 31.3\%) of their total time in quality assurance activities.

Hruschka et al. (2012) propose Quality Driven Software Architecture (QDSA) by putting SA in center of development activities to ensure software products have key quality characteristics of Maintainability, Scalability, and Modularity etc. covered. He stresses that by assigning Quality ownership to SA would result in well negotiated, precise, and quantifiable quality goals that would ensure project goals (ownership of project manager) go hands in hand with Quality goals and conflicts are discussed and resolved proactively.

Karre et al. (2016) proposes heuristic based approaches of Defect dependency, CFGs, and Feature correlation to study defects in large scale integrated software solutions e.g. CBDs. They propose using Defect Dependency Metric to improve overall quality and track defect dependency in such complex systems. Atish et al. (2017), based on similar lines, discusses bipartite relationship between Analysts and CBDs for modelling business systems. Their research concludes that: Analysts develop accurate models by reusing components, and because of their simplicity and compactness, CBDs allow Analysts even with less industry experience perform at par with more experienced ones without effecting quality and costs.

Hareton (1997) stresses that user acceptance testing, where the system is tested in actual usage environment and gathers real time inputs, is another vital cog for success of software projects. Jennifer Biewer (2017) proposes that finding a quality analyst with the business knowledge would be very helpful in successful completion of this crucial stage of the software development. He goes on to say that by teaming up quality analysts with the experienced business analysts we can certainly produce very good results; alternatively we should encourage the integration of these two positions to find optimized results.

Ritesh Kaul (2015) discusses the problems faced by large scale software enterprises where multiple teams which are often geographically sparse work on the same project. He says that while these companies have multiple teams with different quality (at times even conflicting) goals, absence of a central collaboration framework (personnel) often delays effectiveness of agile development timelines. He suggests that by integrating the quality analyst and business analyst roles these companies can resolve Multivendor outsourcing strategies and agile development issues. He stresses that this integration model is much more efficient as it places same person on both ends of the development and helps create better understanding. 


\section{PROPOSAL FOR PROCESS IMPROVEMENT VIA BQA}

\subsection{Definitions and Process}

Industry wide, IT companies continue to employ Business Analysts, System Analysts, Solution Analysts, Business Architects, Solution Architects, and Requirement Engineers etc. for nearly similar roles. While technically distinct definitions exists in literature for each of these roles, practical implications like size of company, kind of business and time/resource constraints often means that all of these roles are often merged into a single person. At their core, these people are responsible to interact with all of the involved stakeholders and bring answer to a key question: What do the systems need to do, and how do they do it?

Hence, by definition, an Analyst has following key roles and responsibilities:

- Interacting with business stakeholders for requirement elicitation, analysis, and prioritization

- Translate and document business needs as functional, non-functional, domain requirements

- Creating design prototypes for requirement, architecture, solution validation

- $\quad$ Act as liaison between in-house developers and outside clients

\subsection{Case Study}

While there are increasing direct/indirect calls of formal role integration of a BQA in Agile framework, no numerical data (as per our findings) currently exists to support potential benefits of such a leap. To bridge this gap, this paper is a small step in that direction. Based in US, Z Technologies (Company name has been kept anonymous to protect confidentiality of data) is a SAAS based multinational software company that deals with Customer Communication Management (CCM) based solutions and services. Over the past 30 years, the company's global clientele includes key players from healthcare, insurance and telecom industry.

The company is a key proponent of Agile framework and uses integration of Confluence with Jira for streamlining of development activities like user stories, sprints, tasks, bug reporting and resolution etc. Because of the nature of their products and services they provide, Analysts in $\mathrm{Z} \mathrm{Tech}$. were found to be practical examples of BQAs. Being in close contact with customers allows them to play dual role of product champions and naysayers, e.g. Quality Engineers or Quality Analysts.

\subsection{Data Analyses}

Based on analysis of Bug reporting/resolution and Enhancement Requests (ERs) data of Z Tech. from 2013-2017, we are in a position to numerically evaluate the benefits of a BQA in Agile framework for large companies that provide SAAS based solutions by coming up with following two hypothesis.

Hypothesis \# 1: Bugs reported by Analysts are critical for product quality and its overall acceptance and hence should receive high priority for defect resolution.

As true representative of client expectations and being both aware of their immediate business needs as well as products current capabilities, Analysts are not only responsible to capture and convey quality characteristics to those involved in development and decision making, but are also responsible to validate (both before and after development) and prioritize these through project managers.

The quality covering efforts they put in include: Regression Testing of customer solutions against each new release, End to End workflow testing ensuring all components are interfaced correctly, Functional Testing including Installation Testing, Relevant Non-functional testing e.g. Performance measures, Localization etc., Root Cause Analyses of customer reported defects.

During these testing efforts - in principal with normal defect reporting/resolution cycle - Analysts report all encountered defects after verification with defect severity, defect priority and acceptance criteria. Defect severity and customer priority are two key factors that determine early resolution of reported defects. Once appropriate corrective actions are implemented by relevant teams, Analysts validates defect correction. 
Table 1. Summary of bugs reporting and fixed

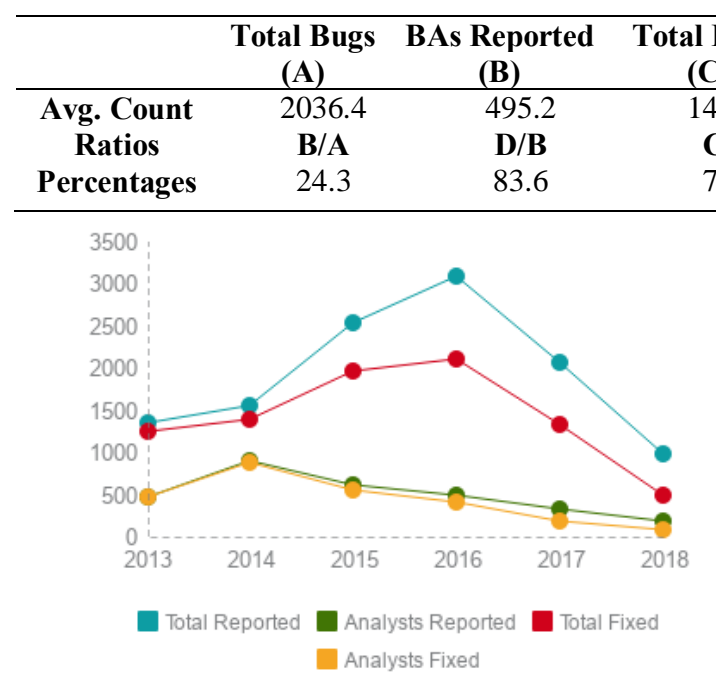

Figure 1. Bugs reported vs. bugs fixed

\begin{tabular}{cccc}
$\begin{array}{l}\text { al Fixed } \\
\text { (C) }\end{array}$ & $\begin{array}{c}\text { BAs Fixed } \\
\text { (D) }\end{array}$ & $\begin{array}{c}\text { Total Not Fixed } \\
(\mathbf{E})\end{array}$ & $\begin{array}{c}\text { Analysts Not Fixed } \\
(\text { F) }\end{array}$ \\
\hline 1449.2 & 413.8 & 587.2 & 81.4 \\
C/A & D/C & E/A & F/B \\
71.1 & 28.6 & 28.8 & 16.4 \\
\hline
\end{tabular}

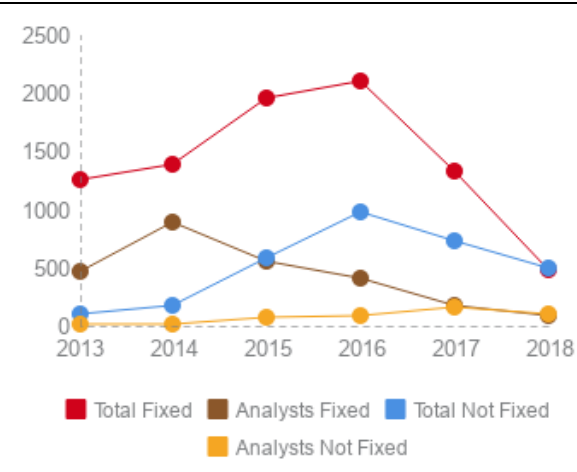

Figure 2. Bugs fixed vs. not fixed

From Table 1and Figures 1 and 2, we can draw following conclusions:

- Analysts are playing a key role in defect reporting and resolution process. As Figure 1. Bugs reported vs. bugs fixed shows, from 2013-2018, they reported nearly a quarter (25\%) of total reported defects $(\mathbf{B} / \mathbf{A})$, and were responsible for resolution of nearly a third (30\%) of total fixed defects (E/A).

- Bugs reported by Analysts are of critical nature for both product quality and acceptance and hence receive higher priority for resolution. From Figure 2. Bugs fixed vs. not fixed, it is evident that while overall Defect Reporting to Defect Fixation ratio $(\mathbf{C} / \mathbf{A})$ is about $71 \%$, the same fixation ratio for Analysts reported defects (D/B) is nearly $84 \%$.

Hypothesis \# 2: ERs reported by Analysts are a representative of customer immediate needs and hence gets prioritized accordingly.

Being in direct contact with customer representatives allows Analysts to have a deeper understanding of client business needs as well as potential pain points. This knowledge helps them translate those business needs into product technological needs and hence identify and prioritize development of key features (for each sprint) by involving relevant stakeholders e.g. Project Manager, Product Owner, Scrum Masters etc.

While traditionally ERs are considered responsibility of Project Managers, putting Analysts in the mix allows them to timely forecast and advocate for customer needs.

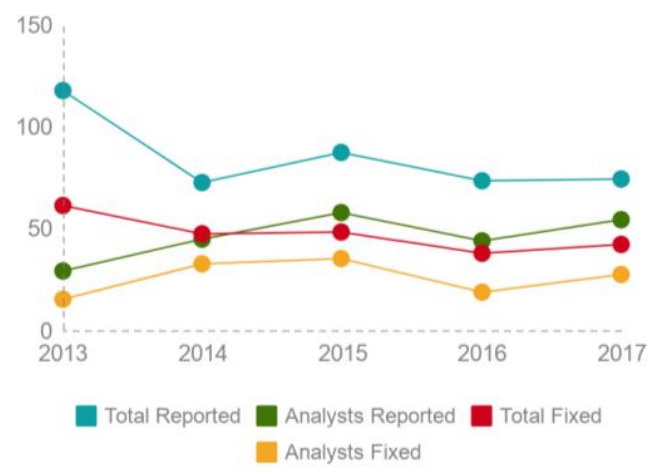

Figure 3. ERs requested vs. fixed report

Table 2. Summary of ERs reported vs. fixed

\begin{tabular}{ccccc}
\hline Years (2013 - 2018) & Total ERs (A) & BAs Reported (B) & Total Fixed (C) & BAs Fixed (D) \\
\hline Avg. Count & 84.6 & 45.4 & 47 & 25.4 \\
Ratios \& Percentages & B/A & $\mathbf{5 3 . 6 6}$ & D/B & $\mathbf{5 5 . 9 4}$ \\
\hline
\end{tabular}


A quick analysis of Figure 3 and Table 2 reveals strong support for above made claims with following key findings:

- As the processes mature, more and more ERs get requested by Analysts. From Figure 3. ERs requested vs. fixed report the decreasing vertical distance between Lines Blue and Green from Years 2013 (29/117) to 2017 (54/74). Overall, Analysts requested for more than half of total reported ERs (B/A) i.e. $54 \%$ during the past 5 years.

- More than half of reported ERs by Analysts (D/B) i.e. 56\% get fixed in earliest Sprints (Line Yellow) as per client's priority. (While rest of these are either deferred for future releases, or are added to backlog for further discussion, while a few gets rejected.)

\section{CONCLUSION}

From the available data and shared hypothesis, it can be argued that with their Business and Technical knowledge, integrating BA and QA into a single role BQA in agile environments, can help large scale enterprises achieve better quality results. A BQA can guarantee successful delivery of quality solutions by proactively taking part in both requirement definitions and validations. A properly elicited set of requirements, with clear cut quality expectations and acceptance metrics, later validated against same established metrics by the same person is bound to buffer information gaps that can otherwise exist between different parties, and eventually help deliver a cost effective, quality focused solution. With focus of IT companies shifting towards large scale, customizable products and service based architecture, BQAs can play an important part in delivery of value based, and quality focused services to clients.

Possible limitations of this study include non-applicability of a BQA in a traditional software development environment and lack of existing data to numerically evaluate benefits of this role integration.

\section{REFERENCES}

Atish P. S.et al, 2017. Reusing business components and objects for modelling business systems: The influence of decomposition characteristics and analyst experience. Journal of Systems and Software, Vol. 131, pages 550-569.

Bennett-Therkildsen A. et al, 2013. Redefinition of the Requirements Engineer Role in Mjølner's Software Development Process. In: Doerr J., Opdahl A.L. (eds) Requirements Engineering: Foundation for Software Quality. Vol. 7830. Springer, Berlin, Heidelberg

Daneva M.et al, 2013. Software Architects' Experiences of Quality Requirements: What We Know and What We Do Not Know? In: Doerr J., Opdahl A.L. (eds) Requirements Engineering: Foundation for Software Quality. Vol. 7830. Springer, Berlin, Heidelberg.

Feldman. S, 2005. Quality Assurance: Much More than Testing. Magazine: Queue - Quality Assurance ,Vol. 3 No. 1, pp. 26-29, ACM, New York, USA.

Hareton K. N.et al, 1997. A study of user acceptance tests. Software Quality Journal, Vol. 6 No. 2, pp 137-149.

Herrmann A, 2013. Requirements Engineering in Practice: There Is No Requirements Engineer Position. In: Doerr J., Opdahl A.L. (eds) Requirements Engineering: Foundation for Software Quality. Vol. 7830. Springer, Berlin, Heidelberg

Hruschka P, 2012. Quality Driven Software Architecture. In: Biffl S., Winkler D., Bergsmann J. (eds) Software Quality. Process Automation in Software Development. Vol. 94. Springer, Berlin, Heidelberg.

Jennifer Biewer. 2017. Value of a Partnership Approach to User Acceptance Testing, https://www.fisglobal.com/

Karre S.A.et al, 2016. Heuristic Approaches to Improve Product Quality in Large Scale Integrated Software Products. In: Maciaszek L.A., Filipe J. (eds) Evaluation of Novel Approaches to Software Engineering. Communications in Computer and Information Science, Vol. 599. Springer, Cham.

Ritesh Kaul, 2015. Integrating BA and QA in Healthcare IT, https://www.emids.com/how-baqa-integration-supportsagile-adoption/

Robin Poston.et al, 2014. Managing User Acceptance Testing of Business Applications, International Conference on HCI in Business, HCIB 2014: HCI in Business, pp 92-102, Springer.

Vinod Kumar Chauhan, 2015. How to reduce user story reopen count in Scrum development? 2nd International Conference on Computing for Sustainable Global Development (INDIACom), pages 2204 - 2208, IEEE. 